\title{
Enseñanza y divulgación de la electrotecnia en España a finales del siglo XIX: las colecciones técnicas de Bailly-Baillière
}

\author{
Teaching and dissemination of electrical engineering in Spain \\ at the end of the $19^{\text {th }}$ century: collections of technical manuals \\ by the publisher Bailly-Baillière
}

José Antonio Moreno Villanueva

Profesor lector Serra Húnter, Universitat Rovira i Virgili. joseantonio.moreno@urv.cat Recibido: 11.05.2019. Aceptado: 06.08.2019

Resumen: A finales del siglo XIX, el interés por las aplicaciones de la electricidad iba más allá de las aulas de las escuelas técnicas y universidades. La comunidad técnica ya no estaba integrada solo por quienes habían seguido estudios de ingeniería, sino también por los electricistas prácticos, que necesitaban asimismo formarse en este ámbito. Entre las obras surgidas con este propósito destacan las dos colecciones publicadas por la editorial Bailly-Baillière e Hijos que son objeto de este estudio: la Pequeña Enciclopedia Electromecánica (1896-1897), dirigida por Henry de Graffigny, y la Biblioteca Completa de Electricidad (1898), de Thomas O'Conor Sloane. Ambas, de carácter marcadamente divulgativo, disfrutaron de una extraordinaria acogida y contribuyeron sin duda a la enseñanza y popularización de la electrotecnia.

Palabras clave: enseñanza técnica; electrotecnia; ciencia popular; colecciones de divulgación; Bailly-Baillière.

\begin{abstract}
At the end of the 19th century, interest in the applications of electricity was beyond the classrooms of technical schools and universities. The technical community no longer consisted solely of those who had studied engineering, but also included practical electricians, who also needed training in this area. Of particular note among the works that emerged to meet this need are two collections published by Bailly-Baillière e Hijos, which are the object of the present study: the Pequeña Enciclopedia Electromecánica (1896-1897), edited by Henry de Graffigny, and the Biblioteca Completa de Electricidad (1898), by Thomas O'Conor Sloane. Both were clearly intended to help spread knowledge of electromechanics and electricity and were very well received. Without a doubt they helped teach and popularise electrical engineering.
\end{abstract}

Keywords: technical education; electrotechnics; popular science; educational collections; Bailly-Baillière.

》 Moreno Villanueva, José Antonio. 2019. “Enseñanza y divulgación de la electrotecnia en España a finales del siglo xix: las colecciones técnicas de Bailly-Baillière". Quaderns de Filologia: Estudis Lingüistics XXIV: 349-375. doi: 10.7203/QF.24.16317 



\section{Introducción ${ }^{1}$}

La historia de la física en España a lo largo del siglo XIX es, sobre todo, la historia de su recepción ${ }^{2}$. Aunque se dieron aportaciones notables en algunos ámbitos, sobre todo en su vertiente aplicada ${ }^{3}$, lo cierto es que los trabajos de autor español, salvo loables excepciones, carecían en general de originalidad y mostraban una clara deuda respecto de las obras francesas. De hecho, como ponían de manifiesto las relaciones de libros obligatorios y recomendados que se publicaron en ese periodo, buena parte de los manuales empleados para su enseñanza en las universidades y las primeras escuelas de ingeniería correspondieron a traducciones de obras francesas, como las de Biot (1826), Beaudant (1839), Despretz (1839), Pouillet (1841) o Ganot (1853), cuando no directamente a textos franceses, como ocurrió con los de Péclet (1832) o Lamé $(1840)^{4}$.

El caso de la electricidad, que experimentó un imparable desarrollo en el último tercio de siglo, hasta convertirse en indudable protagonista de los últimos años de la centuria, no fue una excepción. El impulso que, desde la aparición del Real Decreto de 4 de septiembre de 1850, se quiso dar a las enseñanzas técnicas para estimular el desarrollo industrial no logró acabar con la dependencia de los textos extranjeros, pero sí sirvió para que poco a poco se fueran configurando, entre otros, los estudios de ingeniería industrial ${ }^{5}$. A partir de ese momento, sobre todo

\footnotetext{
${ }^{1}$ Este trabajo se inscribe en el proyecto El léxico especializado en el español contemporáneo, financiado por el Ministerio de Ciencia, Innovación y Universidades (PGC2018093527-B-I00) y desarrollado por el grupo Neolcyt; y en las líneas de trabajo del grupo de investigación en Lexicografía y Enseñanza de Lenguas Extranjeras (LEXELE) de la Universitat Rovira i Virgili, reconocido por la Generalitat de Catalunya como grupo de investigación consolidado (2017 SGR 350).

${ }^{2}$ Para el estudio de la historia de la física en España desde la Ilustración hasta finales del siglo XIX, sigue siendo de referencia el trabajo de Moreno González (1988).

${ }^{3}$ Por lo que respecta a la electricidad, Sánchez Miñana (2006) refiere las aportaciones que se dieron en Barcelona durante la etapa isabelina, en particular entre 1840 y 1860 , de la mano de Pedro Vieta, Juan Agell y Torrents, Francisco Domènech y Maranges, Ambrosio Garcés de Marcilla y Ramón Rosselló, entre otros.

${ }^{4}$ Las fechas indicadas entre paréntesis corresponden a las primeras ediciones en español, salvo en el caso de Péclet y Lamé. Véase Moreno Villanueva (2015).

${ }^{5}$ El volumen de Silva (2007) ofrece una completa aproximación al desarrollo de los estudios de ingeniería industrial en España a lo largo del siglo XIX. Para los propósitos de este artículo, resultan de especial interés la introducción del propio Silva (pp. 7-69),
} 
desde la década de 1870, los ingenieros industriales, particularmente los vinculados a la escuela industrial de Barcelona, iban a tener un papel decisivo en la introducción y el desarrollo de la electrotecnia en España (alumbrado eléctrico, telefonía, etc.) ${ }^{6}$.

A finales de siglo, en los centros oficiales, para la enseñanza de la física industrial y de las aplicaciones de la electricidad se seguían empleando manuales tanto franceses como de autor español -los menos- no siempre suficientemente actualizados. Ahora bien, hay que tener presente que, por esos años, el interés por la electricidad aplicada se extendía fuera de las aulas. En efecto, la comunidad técnica, en un sentido amplio, ya no estaba conformada solo por quienes habían cursado estudios reglados de ingeniería, sino también por los obreros, mecánicos e instaladores electricistas empleados en la naciente industria eléctrica, que necesitaban asimismo formarse en este ámbito. Incluso los particulares sentían la necesidad de conocer con mayor profundidad el funcionamiento de las máquinas y aparatos eléctricos, cada vez más presentes en los establecimientos públicos, los hogares y, en general, la vida cotidiana?

En este contexto, no tardaron en surgir distintas obras de corte divulgativo o vulgarizador dirigidas, por una parte, a los llamados electricistas prácticos $\mathrm{y}$, por otra, al público interesado en los avances en esta materia. A este respecto, es preciso subrayar que los libros y manuales oficiales destinados a la enseñanza de la física y la física industrial, que proporcionaban principalmente sus bases teóricas, encontraron en estos textos de ciencia popular un buen complemento para la enseñanza de la electrotecnia. En España, como venía siendo habitual, las obras fran-

así como las aportaciones de Ramón \& Silva (2007), de Cano Pavón (2007) y de Lusa Monforte (2007).

${ }^{6}$ Tras la supresión de las escuelas industriales de Gijón y Vergara (1860), la de Valencia (1865) y la de Madrid (1866), entre 1867 y 1899 -fecha de la creación de la escuela de Bilbao-, la Escuela Superior de Ingenieros Industriales de Barcelona fue la única institución de estas características en toda España. Los trabajos de Lusa Monforte (2002, 2007) y Alayo \& Sánchez Miñana (2011) ofrecen un panorama de las aportaciones de la escuela barcelonesa. Asimismo, resulta de interés la obra de Alayo (2007), dedicada a la introducción de la electricidad en Cataluña entre 1875 y 1935.

${ }^{7}$ La tesis de Ferran Boleda (2013), aunque centrada en el periodo 1929-1936, ofrece una buena aproximación a los públicos de la electricidad y, en general, al proceso de comunicación y divulgación de la iluminación eléctrica y de los aparatos eléctricos para uso doméstico en el contexto catalán. 
cesas, originales o traducidas, fueron el referente indiscutible, sin que por ello se deba dejar de considerar la creciente presencia de autores ingleses y americanos y, por supuesto, la aportación de los españoles.

En este trabajo, en particular, se profundiza en el estudio de dos colecciones publicadas por la editorial Bailly-Baillière e Hijos, correspondientes a sendas traducciones del francés y el inglés, que disfrutaron de un extraordinario éxito en el cambio de siglo y que contribuyeron sin duda a la popularización de la electrotecnia, así como a su enseñanza en las escuelas de ingeniería y, sobre todo, en las escuelas técnicas especiales y de artes y oficios: la Pequeña Enciclopedia Electromecánica, dirigida por Henry de Graffigny, y la Biblioteca Completa de Electricidad, de Thomas O'Conor Sloane.

\section{Bailly-Baillière y la divulgación de la ciencia y la técnica en España}

La librería casa editorial Bailly-Baillière, establecida en Madrid en 1848, disfrutó de un notable protagonismo durante la segunda mitad del siglo XIX y los primeros años del siglo XX gracias a la extraordinaria labor de difusión del conocimiento científico que llevó a cabo en ese periodo, favorecida, entre otras razones, por su estrecho contacto con las librerías francesas ${ }^{8}$. Así lo han destacado distintos trabajos, como los de Botrel (1988, 1993) y Martínez Marín (2001) y, singularmente, los de Riera \& Riera (2005) y Simon (2006, 2010), que han profundizado en la decisiva aportación de Carlos Bailly-Baillière y sus sucesores por lo que respecta a la introducción en España de obras de medicina y física, respectivamente.

Siguiendo la línea abierta por esos estudios, en Moreno Villanueva (2017a) se incide en el papel que Bailly-Baillière desempeñó en relación con la importación de colecciones de divulgación científica, un género hasta entonces todavía poco explorado en el panorama editorial español ${ }^{9}$. En efecto, tras la muerte de su fundador en 1892, la editorial,

\footnotetext{
${ }^{8}$ Entre esas librerías se deben destacar las vinculadas a la familia Baillière, que conformaba una extensa red internacional de editores, lo que facilitaba la circulación de los títulos de sus catálogos. Véase Gourevitch \& Vincent (2006) y Simon (2007).

${ }^{9}$ Constituye una notable excepción la Biblioteca Enciclopédica Popular Ilustrada, publicada en Madrid por Gregorio Estrada entre 1877 y 1889, que se acompañó de la edición paralela de la Revista Popular de Conocimientos Útiles, en cuya redacción
} 
ya en manos de Enrique y Antonio Bailly-Baillière, apostó de manera decidida por la edición de pequeñas bibliotecas de "libros populares de consulta" -así se presentaban en los anuncios incluidos en otras publicaciones de la casa editorial-destinados a satisfacer el interés que los avances técnicos y científicos despertaban entre el creciente número de prácticos y aficionados. Así se ponía de manifiesto en la siguiente noticia ("La Casa Bailly-Bailliére é hijos") aparecida en el Heraldo de Madrid del 12 de junio de 1907 con motivo del inicio de la publicación de la Nueva Biblioteca de Autores Españoles, dirigida por Marcelino Menéndez y Pelayo:

La historia de esta conocida Casa editorial es la del desenvolvimiento científico de nuestra patria desde la primera mitad del siglo XIX. [...]

Entre los libros comprendidos en estos catálogos citaremos sus conocidas obras de vulgarización, como el popular Almanaque ó Pequeña Enciclopedia de la vida práctica, sus Agendas culinaria y de bufete, sus notables Pequeñas Enciclopedias prácticas de electricidad, de electromecánica, de construcción, del aprendiz y aficionado electricista, de Química industrial, del fotógrafo aficionado, de agricultura y de viticultura y vinicultura.

Todas las colecciones citadas, consagradas al estudio de una determinada parcela técnica o científica, se editaron a partir de 1896 y disfrutaron de una excelente acogida entre un amplio público, como muestra el hecho de que la mayor parte de sus volúmenes contaran con varias tiradas hasta entrada la segunda década del siglo XX. Entre ellas, además de las dos colecciones que son objeto del presente estudio, figura el Manual del Aprendiz y Aficionado Electricista, también de Henry de Graffigny; con todo, solo tengo noticia de la publicación de su quinto tomo, titulado Alumbrado eléctrico de las habitaciones y edificios, traducido por Ricardo Yesares Blanco y que en las páginas de La Energía Eléctrica (10-IV-1907: 136) se presentaba, no por casualidad, como un libro "Para los electricistas y cuantas personas tengan luz eléctrica en sus casas".

participaron los autores de la citada colección. Entre sus 101 tomos figuran el Manual de física popular (1878) de Gumersindo Vicuña y, sobre todo, el Manual de electricidad popular (1881) de José Casas Barbosa, una de las primeras obras de carácter divulgativo de autor español dedicada al estudio de la electricidad. 
Entre las razones que explican el éxito de estas colecciones de divulgación figuran, en primer lugar, el módico precio de sus volúmenes, su fácil manejo y su sencilla pero cuidada edición, manifiesta tanto en la cantidad como en la calidad de los grabados. Por otra parte, no hay que perder de vista que la editorial Bailly-Baillière, a través de su extensa red de corresponsales, desplegó una intensa campaña de publicidad tanto en las revistas de carácter científico como en los diarios nacionales y regionales, en cuyas páginas se publicaron reseñas que destacaban la claridad de la exposición, la calidad de la traducción y, especialmente, su utilidad.

Ahora bien, como apunta Béguet (1990: 7), “cette médiation qu'est la vulgarisation présuppose [...] la disposition d'un large public à recevoir les rudiments de la connaissance scientifique [...] en outre l'existence de conceptions scientifiques clairement affirmés". En España, por lo que respecta a la electricidad, esas condiciones se dieron solo a partir del último cuarto del siglo XIX, cuando, gracias a la labor desplegada por los ingenieros industriales, el desarrollo de la electrotecnia pudo seguir los pasos del resto de Europa (Lusa Monforte, 2003; Alayo \& Sánchez Miñana, 2011). De hecho, se puede afirmar, siguiendo al citado autor francés, que el discurso vulgarizador encontró el mejor aliado en la industrialización, en este caso en la joven industria eléctrica, pues la omnipresencia de las aplicaciones de la electricidad en muy diversos ámbitos hizo nacer un clima favorable a la instrucción de la población en esta materia.

En otras palabras, la necesidad de transmitir los conocimientos técnicos y científicos más allá de las aulas de las escuelas técnicas y de artes y oficios, aunque también en ellas; la necesidad de enseñar no solo a los ingenieros eléctricos, sino también a los llamados electricistas prácticos, esto es, a los obreros y trabajadores empleados en fábricas y talleres, e incluso a los usuarios particulares, revistió a los manuales de electricidad popular del carácter de instrumentos de utilidad social, al servicio del progreso del conjunto de la sociedad.

Es evidente que la editorial Bailly-Baillière, consciente de la carencia de textos destinados a cubrir esa necesidad, supo aprovechar la oportunidad que se abría en el panorama editorial y apostó por introducir en España, además de las colecciones que son objeto de este estudio, algunos de los títulos franceses e ingleses sobre electricidad que contaron con mayor difusión por aquellos años. Así lo destacaba la siguiente 
nota bibliográfica aparecida en El País (12-IX-1897: 3), coincidiendo con la aparición de los últimos volúmenes de la Pequeña Enciclopedia Electromecánica:

Un aplauso muy sincero y justo merecen los Sres. Bailly-Bailliére é Hijos por la difícil empresa que acaban de terminar, según nuestras noticias con bastante fortuna. No nos extraña, pues quien ha llevado á cabo la publicación de obras tan importantes como el Diccionario de Electricidad y Magnetismo, de Lefevre; el Manual del Constructor, de Soroa y Castro; la Guía práctica de Electricidad industrial, de Dumont, y Propiedades fundamentales de las corrientes alternas, simples y polifásicas, de Peña y Braña, bien puede acometer la ruda tarea de publicar una obra de doce tomos, ilustrada con copiosos y magníficos dibujos y $[\ldots]$ venderla á un precio sumamente ínfimo $[\ldots]$.

\section{La Pequeña Enciclopedia Electromecánica}

Los doce volúmenes que integran la Pequeña Enciclopedia Electromecánica vieron la luz entre finales de 1896 y julio de 1897, con apenas unos meses de diferencia respecto a los títulos de la edición francesa, lo que evidencia la estrecha relación que mantuvo Bailly-Baillière con las casas editoriales parisinas, en este caso con la Librairie Scientifique et Industrielle des Arts et Manufactures E. Bernard et Cie., bajo cuyo sello se editó la Petite Encyclopédie Electro-Mécanique. De hecho, la traducción española conservó buena parte de las características de la edición francesa: el formato en octavo, los grabados y, en parte, la presentación, pues la cubierta de la encuadernación en rústica reprodujo su característica cenefa y los volúmenes encuadernados en tela eran asimismo de color rojizo, con nervios y caracteres dorados.

Su director, Henry de Graffigny (1863-1934), fue uno de los principales exponentes de la segunda generación de vulgarizadores franceses, que siguieron la senda abierta entre 1860 y 1890 por Louis Figuier, Victor Fonvielle, Amedée Guillemin, Henri de Parville, Camille Flammarion y Gaston Tissandier, entre otros (Béguet, 1990: 7-15). Autor de más de doscientos títulos sobre ciencias aplicadas, astronomía, aeronáutica, mecánica, química, radiología y, por supuesto, electricidad, escribió asimismo algunos libros de viajes y novelas, y desarrolló una intensa actividad como periodista científico al frente de publicaciones 
como el Journal des Inventeurs, la Revue des Inventions Techniques o La Science Universelle ${ }^{10}$.

La Petite Encyclopédie Electro-Mécanique, que la casa francesa no dudó en calificar de "véritable révolution en librairie"11, se publicitó en los siguientes términos en Bibliographie de la France. Journal général de l'imprimerie et de la librairie (n. ${ }^{\circ} 25$, enero de 1896: 160):

Cette collection [...] constituera le plus précieux vade-mecum, la bibliotèque la plus complète et la plus nécessaire à tous les ingénieurs, directeurs de stations centrales pour l'éclairage ou le transport de l'électricité, ouvriers monteurs et poseurs de sonnettes et téléphones, galvanoplastes, nickeleurs, chauffeurs et conducteurs de machines à vapeur, à gaz ou à pétrole, amateurs, enfin à toutes les personnes qui s'intéressent, théoriquement ou pratiquement, aux applications de l'électricité et de la mécanique.

Tales palabras se reprodujeron casi literalmente en la publicidad que daba noticia de su edición en español en las páginas de las publicaciones periódicas de la época. En la misma línea se expresaba su director, Graffigny, en el prólogo al primero de los volúmenes ${ }^{12}$ :

Sin duda alguna existen $[\ldots]$ numerosos tratados de electricidad industrial. [...] el electricista que quiera formar una biblioteca técnica tiene ancho campo donde elegir obras que traten de electricidad.

Pero el electricista práctico que busque un formulario de uso constante, el obrero que desee instruirse, el aficionado que necesite una colección de notas claras y precisas, todos los que necesiten, en una palabra, un guía serio, encontrarán sin duda generalmente muchas dificultades para formar dicha biblioteca (Graffigny, 1898, vol. 1: 1-2).

Esas dificultades, según Graffigny, eran fundamentalmente de dos tipos. Por una parte, las obras existentes eran "incompletas", en el sentido de que no todos los interesados podían encontrar en ellas lo que

\footnotetext{
${ }^{10}$ Bailly-Baillière publicó otros tres títulos de este autor relacionados con la electricidad: Elementos de electricidad general (1906), traducida por José María de Soroa y Enrique de Pineda, y Todo el mundo electricista (1912) y Formulario del obrero mecánico, electricista, automovilista, constructor, aviador (1913), estas últimas traducidas por Ricardo Yesares Blanco (Moreno Villanueva, 2017a).

${ }^{11}$ Catálogo del año 1900 de la editorial E. Bernard et Cie. (p. 33).

${ }^{12}$ En adelante cito siempre por la edición española de la colección.
} 
buscaban; dicho de otro modo, no servían "más que para cierto número de lectores" (ibid.: 2). Por otra parte, si se pretendía reunir los variados manuales técnicos y aplicados publicados hasta la fecha sobre la ciencia eléctrica, era preciso invertir una importante suma de dinero.

En la figura 1 se ofrece el detalle de los doce volúmenes que conforman la colección. La mayoría de ellos contaban con cerca de 160 páginas profusamente ilustradas con dibujos intercalados en el texto (solo los volúmenes 3 y 4, con 146 y 132 páginas, respectivamente, eran de menor extensión). Su precio era de 1,5 o 2 pesetas, según se adquirieran encuadernados en rústica o en tela a la inglesa; la colección completa en rústica se vendía a 18 pesetas, y en tela, a 24 pesetas.

\begin{tabular}{|l|l|l|l|l|}
\hline \multicolumn{1}{|c|}{ Título francés (1896) } & \multicolumn{1}{|c|}{$\begin{array}{c}\text { Título del volumen } \\
\text { (1896-1897) }\end{array}$} & \multicolumn{1}{|c|}{ Traductor } & \multicolumn{1}{c|}{ Tiradas $^{13}$} & Figuras \\
\hline $\begin{array}{l}\text { 1.- Manuel élémentaire } \\
\text { d'électricité industrielle }\end{array}$ & $\begin{array}{l}\text { 1.- Manual elemental de } \\
\text { electricidad industrial }\end{array}$ & $\begin{array}{l}\text { Ramón Cases } \\
\text { Civera }\end{array}$ & $\begin{array}{l}1896,1897, \\
1902,1903\end{array}$ & 68 figs. \\
\hline $\begin{array}{l}\text { 2.- Manuel du conducteur } \\
\text { de dynamos et moteurs } \\
\text { électriques }\end{array}$ & $\begin{array}{l}\text { 2.- Manual práctico del } \\
\text { encargado de dinamos y } \\
\text { motores eléctricos }\end{array}$ & $\begin{array}{l}\text { Luis de la Peña } \\
\text { y Braña }\end{array}$ & $\begin{array}{l}1896,1899, \\
1903\end{array}$ & 69 figs. \\
\hline $\begin{array}{l}\text { 3.- Les piles et les accumu- } \\
\text { lateurs }\end{array}$ & 3.- Pilas y acumuladores & $\begin{array}{l}\text { Ramón Escan- } \\
\text { dón }\end{array}$ & $\begin{array}{l}1896,1897, \\
1899,1902, \\
1903,1904\end{array}$ & 69 figs. \\
\hline $\begin{array}{l}\text { 4.- Les canalisations } \\
\text { électriques }\end{array}$ & $\begin{array}{l}\text { 4.- Las canalizaciones } \\
\text { eléctricas }\end{array}$ & Ramón Cases & 1897,1898, & 40 figs. \\
Civera & 1899,1902, & 1903 \\
\hline $\begin{array}{l}\text { 5.- Chauffeur-conducteur } \\
\text { de machines à vapeur }\end{array}$ & $\begin{array}{l}\text { 5.- Fogonero-conductor } \\
\text { de máquinas de vapor }\end{array}$ & Mauricio Max & 1897,1903 & 47 figs. \\
\hline $\begin{array}{l}\text { 6.- Conducteur de moteurs } \\
\text { à gaz et à pétrole }\end{array}$ & $\begin{array}{l}\text { 6.- El conductor de } \\
\text { motores de gas y de } \\
\text { petróleo }\end{array}$ & León Soulerat & 1897,1898, & 51 figs. \\
\hline $\begin{array}{l}\text { 7.- Guide pratique d'éclai- } \\
\text { rage électrique }\end{array}$ & $\begin{array}{l}\text { 7.- Guía práctica del } \\
\text { alumbrado eléctrico }\end{array}$ & $\begin{array}{l}\text { Ramón Cases } \\
\text { Civera }\end{array}$ & $\begin{array}{l}1897,1898, \\
1899,1903\end{array}$ & 64 figs. \\
\hline $\begin{array}{l}\text { 8.- Le monteur-appareil- } \\
\text { leur électricien }\end{array}$ & $\begin{array}{l}\text { 8.- El montador elec- } \\
\text { tricista }\end{array}$ & $\begin{array}{l}\text { Ramón Cases } \\
\text { Civera }\end{array}$ & $\begin{array}{l}1897,1898, \\
1902,1903\end{array}$ & 103 figs. \\
\hline $\begin{array}{l}\text { 9.- Transport électrique } \\
\text { des forces motrices }\end{array}$ & $\begin{array}{l}\text { 9.- El transporte eléc- } \\
\text { trico de las fuerzas } \\
\text { motoras }\end{array}$ & León Soulerat & $\begin{array}{l}1897,1899, \\
1901\end{array}$ & 46 figs. \\
\hline
\end{tabular}

${ }^{13}$ Se indican, sin pretensión de exhaustividad, las tiradas localizadas en distintos catálogos.

${ }^{14}$ Además de las sucesivas tiradas de la primera edición de este volumen, tengo noticia de la publicación de una segunda edición, fechada en 1907, cuya traducción se confió a Ricardo Yesares Blanco. 


\begin{tabular}{|l|l|l|l|l|}
\hline \multicolumn{1}{|c|}{ Título francés (1896) } & \multicolumn{1}{|c|}{$\begin{array}{c}\text { Título del volumen } \\
(\mathbf{1 8 9 6 - 1 8 9 7 )}\end{array}$} & \multicolumn{1}{|c|}{ Traductor } & \multicolumn{1}{|c|}{ Tiradas $^{13}$} & Figuras \\
\hline $\begin{array}{l}\text { 10.- Les réseaux télépho- } \\
\text { niques et sonnettes }\end{array}$ & $\begin{array}{l}\text { 10.- Redes telefónicas y } \\
\text { campanillas }\end{array}$ & $\begin{array}{l}\text { Ramón Cases } \\
\text { Civera }\end{array}$ & $\begin{array}{l}1897,1899, \\
1902,1903\end{array}$ & 77 figs. \\
\hline $\begin{array}{l}\text { 11.- Guide pratique de } \\
\text { l'électro-chimiste }\end{array}$ & $\begin{array}{l}\text { 11.- Manual del electro- } \\
\text { químico }\end{array}$ & León Soulerat & 1897,1905 & 35 figs. \\
\hline 12.- L'électricité pour tous & $\begin{array}{l}\text { 12.- La electricidad para } \\
\text { todos }\end{array}$ & León Soulerat & 1897,1903 & 78 figs. \\
\hline
\end{tabular}

Figura 1. Volúmenes de las ediciones francesa y española de la Pequeña Enciclopedia Electromecánica

La traducción al español de los tres primeros volúmenes, publicados a finales de 1896, se confió a los ingenieros Ramón Cases Civera, Luis de la Peña y Braña y Ramón Escandón. Todos ellos habían colaborado ya con Bailly-Baillière con anterioridad: Cases Civera había traducido la Guía práctica de electricidad industrial. Alumbrado y transmisiones eléctricas (1896), de Georges Dumont y Gustave Baignères; De la Peña y Braña ${ }^{15}$, miembro de la Institution of Electrical Engineers de Londres, fue autor de Propiedades fundamentales de las corrientes alternas, simples y polifásicas (1893); Escandón, por su parte, se había ocupado de la revisión y ampliación del Tratado elemental de fisica experimental y aplicada y de meteorología de Adolphe Ganot, en sus ediciones 10. ${ }^{\mathrm{a}}$ (1891) a 17. a , traducidas por Eduardo Sánchez Pardo.

En los tomos 4 a 6 se sumaron a Cases Civera los nombres de Mauricio Max y León Soulerat, de quienes apenas constan datos, salvo que el último de ellos era ingeniero de minas. Probablemente, la necesidad de cumplir con el ritmo de publicación previsto -un volumen al mesobligó a la editorial a contar con cinco traductores distintos para los seis primeros volúmenes de la colección. Los seis títulos restantes, finalmente, fueron traducidos por Cases Civera y Soulerat.

Las revistas científicas españolas de la época, tal como había sucedido con la edición francesa, no tardaron en hacerse eco de su publicación. Entre ellas cabe destacar la Revista Minera, Metalúrgica y de Ingeniería, que en su número 1640, de 8 de julio de 1897, daba ya por

${ }^{15}$ Luis de la Peña y Braña fue redactor jefe de Madrid Científico -inicialmente subtitulada Revista de ciencia, ingeniería y electricidad- entre 1894 y 1896 y tradujo el Vademécum del mecánico. Estudios sobre construcción de máquinas, de Armengaud Ainé (1898). 
completada la colección: "Con estos dos volúmenes queda completa la serie de los 12 que constituyen la pequeña enciclopedia electro-mecánica, publicada con general aceptación por la librería editorial de los Sres. Bailly-Baillière é Hijos" (p. 257) ${ }^{16}$. En los mismos términos se expresaba La Época (n. ${ }^{\circ}$ 16987, 19-IX-1897: 4), que refería la edición de nuevas tiradas:

Gran empresa es la que acaban de dar por terminada los Sres. BaillyBaillière é hijos. La utilidad que con ella reportan á cuantos interesan las distintas aplicaciones del fluído eléctrico es incontestable, como lo demuestran las ya repetidas tiradas que se han visto precisados á efectuar de ella apenas terminada su publicación [...].

Las alusiones al carácter práctico de la obra y a su utilidad, así como a la claridad y concisión del texto, fueron recurrentes en las notas bibliográficas aparecidas en la prensa general y científica. Sirvan como muestra los siguientes fragmentos:

El tomo cuarto de esta útil publicación [...] es un resumen completo de cuanto necesitan los electricistas de todas categorías, desde el ingeniero que hace los proyectos hasta el último operario que trabaja en las instalaciones. El estilo claro y conciso con que está escrito, hace del librito uno de muchos informes con relación al número de sus páginas (Revista Minera, Metalúrgica y de Ingeniería, 1-IV-1897: 103).

La sencillez de su lenguaje, el plan seguido por el autor, que no era otro que hacer una obra muy completa y sumamente útil, y los abundantes detalles en ella amontonados con orden admirable, la hacen de utilidad para cuantos se dedican al empleo de la electricidad (La Época, n. ${ }^{\circ}$ 16987, 19-IX-1897: 4).

El propio Graffigny, en los prólogos a los distintos volúmenes, subrayaba la practicidad y utilidad de su Enciclopedia como uno de sus principales valores:

\footnotetext{
${ }^{16}$ La Revista Minera, Metalúrgica y de Ingeniería dio noticia puntual de la aparición de los distintos volúmenes de la colección (n. ${ }^{\circ} 1627,1-\mathrm{IV}-1897$ : 103; n. ${ }^{\circ} 1631,1-\mathrm{V}-1897$ : 135; n. ${ }^{\circ} 1635,1-V I-1897: 167$; n. ${ }^{\circ} 1636,8-V I-1897: 175 ;$ n. ${ }^{\circ} 1638,24-V I-1897: 191$; n. $\left.{ }^{\circ} 1640,8-V I I-1897: 207\right)$. También aparecieron reseñas en El Pallaresa (12-V-1897), Blanco y Negro (3-VII-1897: 18), La Época (n. ${ }^{\circ}$ 16987, 19-IX-1897: 4), Industria e Invenciones (20-XI-1897: 195) o la Revista de Obras Públicas (1897, t. II).
} 
[Esta colección] deberá consultarse, dentro de poco tiempo, por todos los que se dediquen á esta ciencia, lo mismo sobre la mesa del ingeniero de una estación central que en la biblioteca de los propietarios de máquinas de vapor ó de gas, ya en los talleres de galvanoplastia ó, por fin, sobre el banco del ajustador mecánico ó electricista. [...] Si nuestro trabajo reporta $u t_{i l i d a d}{ }^{17}$ á nuestros lectores, cumpliremos el fin propuesto y quedaremos satisfechos (Graffigny, vol. 1, 1896: 4).

Entre los medios dispuestos para servir a ese propósito ocupan un lugar destacado, como venía siendo habitual en las obras de orientación didáctica, las ilustraciones incluidas en el conjunto de la obra-se contabilizan cerca de ochocientas-, "multitud de figuras explicativas que contribuirán seguramente á la rápida inteligencia del texto" (Graffigny, vol. 2, 1896: 3) y que debían facilitar su estudio.

También con fines didácticos, Graffigny decidió ofrecer al término del último volumen de la Pequeña Enciclopedia Electromecánica un breve "Vocabulario del electricista" destinado a reunir "según el orden alfabético, por ser el más sencillo, las varias palabras técnicas que forman parte del lenguaje corriente de los electricistas" (vol. 12, 1897: 112). En él, a lo largo de trece páginas (pp. 112-125), se reúnen 87 tecnicismos pensando en "los aficionados y [...] las personas poco enteradas de las expresiones usadas en la ciencia y la industria eléctricas". Entre los términos incluidos ocupan un lugar destacado las voces relativas a la electrometría y, sobre todo, a la electrotecnia ${ }^{18}$. Como se puede comprender, se trata de un vocabulario parcial, según subrayaba la reseña aparecida en la Revista Minera, Metalúrgica y de Ingeniería (n. ${ }^{\circ} 1640,8-V I I-1897: 257$ ), que lo calificaba de "muy incompleto, y no siempre castizo" ${ }^{19}$. Ello no obstaba para que se destacara su valor como instrumento de divulgación:

\footnotetext{
${ }^{17}$ La cursiva pertenece al original.

${ }^{18}$ Por lo que respecta a la electrometría, figuran ampere, amperimetro, contador, farad, galvanómetro, ohm, volt, vóltmetro, watt y wáttmetro, entre otros; en cuanto a la electrotecnia, se pueden citar alternador, canalización ó circuito, colector, compound, conmutador, dinamo, distribución, electrógeno, filamento, shunt y transformadores. La mayor parte de estos términos no figuraban en los diccionarios generales, entre ellos el de la Real Academia Española.

${ }^{19}$ En otros casos, esta misma revista había puesto de manifiesto el cuidado en la traducción de los términos técnicos: "La competencia de los traductores se demuestra en
} 
Los Sres. Bailly-Baillière pueden estar satisfechos de haber popularizado con la traducción de los 12 tomos de Graffigny las principales cuestiones de electricidad, en la forma y extensión conveniente para la gran masa de aficionados y de interesados en sus múltiples aplicaciones.

El volumen se cerraba con una relación de centrales eléctricas de España -"bastante completa", a juicio del autor de la reseña-, que se extendía en forma de apéndice a lo largo de casi cuarenta páginas (pp. 126-163). Organizada por provincias, en ella se detallan las poblaciones en que se hallaban enclavadas las centrales, así como los pueblos a que suministraban electricidad; los propietarios y directores; los motores y dinamos instalados y su potencia, además de la casa constructora. Se trata de una de las escasas referencias a España que se ofrecen a lo largo de la colección, cuya traducción sigue en todo momento de cerca la edición francesa.

\section{La Biblioteca Completa de Electricidad}

A principios de 1898, tan solo unos meses después de publicar la primera edición en español de la Pequeña Enciclopedia Electromecánica, la editorial Bailly-Baillière inició la publicación de una nueva colección de divulgación destinada asimismo a los electricistas prácticos $\mathrm{y}$, en general, al público interesado por las aplicaciones de la electricidad: la Biblioteca Completa de Electricidad, de Thomas O'Conor Sloane (1851-1940). En esta ocasión se trataba de una colección formada por cinco volúmenes cuyas primeras ediciones en inglés vieron la luz en Nueva York entre 1891 y 1894, de la mano de la editorial Norman W. Henley \& Co. Así pues, aunque su publicación precedió en el tiempo a la obra de Graffigny, fue esta última la primera en contar con una traducción al español. Por otra parte, como se verá después, los títulos de la edición española no aparecieron en el mismo orden en que lo hicieron originalmente.

O'Conor Sloane, formado en la Universidad de Columbia, donde se doctoró en ingeniería eléctrica en 1872, desarrolló una intensa actividad de divulgación científica en las primeras décadas del siglo XX. Fue editor de Scientific American y The Electrical Experimenter, editor aso-

el esmero que han puesto en la tecnología de las obras mencionadas" (Revista Minera, Metalúrgica y de Ingeniería, n. ${ }^{\circ}$ 1631, 1-V-1897: 135). 
ciado de Science and Invention y formó parte del equipo editorial, entre otras, de Everyday Engineering Magazine, que alcanzó una notable popularidad. Sin embargo, fue conocido sobre todo por su participación en Amazing Stories, la primera revista dedicada a la ciencia ficción, fundada en 1926 por Hugo Gernsback y al frente de la cual permaneció Sloane entre 1929 y $1938^{20}$.

A ese mismo propósito divulgativo respondían sus manuales técnicos, entre los que se incluyen los que integran la Biblioteca Completa de Electricidad, que fueron utilizados durante años como libros de texto, como prueban sus numerosas ediciones y reediciones hasta bien entrado el siglo XX. Así, en 1917, a propósito del primero de sus volúmenes, Arithmetic of Electricity, se podía leer lo siguiente en el Catalogue of Latest and Best Mechanical, Scientific and Practical Books - Practical Books for Practical Men de The Norman W. Henley Publishing Co.: "This book is classed among the most useful works published on the science of electricity" 21 .

En la figura 2 se presentan los títulos en inglés -que la editorial neoyorquina presentó ya como "An Electrical Library"-, ordenados por fecha de aparición, y se indican las ediciones de que he tenido noticia.

\begin{tabular}{|l|c|l|}
\hline Título & 1. $^{\text {a }}$ ed. & Ediciones $^{22}$ \\
\hline $\begin{array}{l}\text { Arithmetic of Electricity. } \\
\text { A manual of electrical calculations by } \\
\text { arithmetical methods }\end{array}$ & 1891 & $\begin{array}{l}1895\left(4 .{ }^{\mathrm{a}}\right), 1899\left(5 .{ }^{\mathrm{a}}\right), 1903 \\
\left(16 .{ }^{\mathrm{a}}\right), 1909\left(20 .^{\mathrm{a}}\right), 1915\end{array}$ \\
\hline
\end{tabular}

\footnotetext{
${ }^{20}$ Para más detalle sobre esta revista y la participación de O'Conor Sloane, véanse las respectivas entradas en The Encyclopedia of Science Fiction: $<$ http://www.sf-encyclopedia.com/entry/amazing > [Acceso 20/09/2019].

${ }^{21}$ El catálogo se incluye al término del Glossary of Aviation Terms - Termes d'Aviation compilado por Victor W. Pagé y Paul Montariol y publicado por Norman W. Henley \& Co en 1917.

${ }^{22}$ En el pie editorial del primero de los volúmenes (Arithmetic of Electricity) figura desde la primera edición, además de Norman W. Henley \& Co., la editorial londinense E. \& F. N. Spon. Por otra parte, las ediciones de 1899 de How to become a Successful Electrician y Electric Toy Making for Amateurs corrieron a cargo de Munn \& Co., también de Nueva York, con el copyright de Henley \& Co.

${ }^{23}$ Se trata sin duda del primer volumen de la colección, pues en él se indica que se hallan en preparación los títulos Electricity Simplified y Electric Toy Making.
} 


\begin{tabular}{|c|c|c|}
\hline Título & 1. a ed. & Ediciones $^{22}$ \\
\hline $\begin{array}{l}\text { Electricity Simplified. } \\
\text { The practice and Theory of Electricity. } \\
\text { Including a Popular Review of the The- } \\
\text { ory of Electricity, with Analogies and } \\
\text { Examples of its Practical Applications } \\
\text { in Every-Day Life }\end{array}$ & 1891 & $\begin{array}{l}1895\left(3 .^{\mathrm{a}}\right), 1897\left(6 .^{\mathrm{a}}\right), 1901 \\
\left(10 .^{\mathrm{a}}\right), 1905\left(12 .^{\mathrm{a}}\right), 1911 \\
\left(13 . .^{\mathrm{a}}\right), 1920\left(15 .^{\mathrm{a}}\right)\end{array}$ \\
\hline $\begin{array}{l}\text { Electric Toy Making for Amateurs } \\
\text { Including Batteries, Magnets, Motors, } \\
\text { Miscellaneous Toys and Dynamo Con- } \\
\text { struction }\end{array}$ & 1891 & $\begin{array}{l}1893\left(2 .^{a}\right), 1897\left(3 .^{a}\right) \\
1899,1903,1915\left(20 .^{a}\right) \\
1923\left(21 .^{a}\right)\end{array}$ \\
\hline $\begin{array}{l}\text { The Standard Electrical Dictionary } \\
\text { A popular dictionary of words and terms } \\
\text { used in the practice of electrical engi- } \\
\text { neering }\end{array}$ & 1892 & $\begin{array}{l}1897 \text { ( } 2 .^{\mathrm{a}} \text { ed.), } 1898 \text { (2. } \\
\text { ed., Londres: Crosky Lock- } \\
\text { wood and Son), } 1903 \text { (10. }{ }^{\mathrm{a}} \\
\text { ed.), } 1924 \text { (ed. adicionada } \\
\text { por Arthur E. Watson) }\end{array}$ \\
\hline $\begin{array}{l}\text { How to become a Successful Electrician } \\
\text { The Studies to be Followed, Methods of } \\
\text { Work, Fields of Operation, Professional } \\
\text { Ethics and Wise Counsel }\end{array}$ & 1894 & $\begin{array}{l}1899,1901\left(9 .^{a}\right), 1903 \\
\left(12 .^{a}\right), 1906\left(15 .^{a}\right), 1913 \\
\left(18 .^{a}\right)\end{array}$ \\
\hline
\end{tabular}

Figura 2. Títulos en inglés de los volúmenes de la Biblioteca Completa de Electricidad

La traducción al español de los cinco volúmenes corrió a cargo de José Pla, de quien no constan otros datos ${ }^{24}$. Sin duda, el éxito cosechado por la Pequeña Enciclopedia Electromecánica llevó a la editorial BaillyBaillière a apostar por incorporar a su catálogo una colección que guardaba notables similitudes con la obra de Graffigny, tanto por lo que respecta a su contenido como en cuanto a su presentación, pues se editó

\footnotetext{
${ }^{24}$ Probablemente se trata del mismo José Pla que tradujo del francés la novela El amo del mar (1905), del vizconde E. M. de Vogüe. A este respecto, Emilio Huguet del Villar, en el artículo "La industria del libro" aparecido en Nuevo Mundo (n. ${ }^{\circ}$ 593, 18-V-1905: 3), apuntaba: "varias empresas editoriales de Madrid y provincias, sobre todo de las ricas provincias del Este, han encontrado una verdadera mina en las traducciones. [...] Una de las mejores traducciones, o por lo menos de las menos censurables, que me han caído en las manos de algún tiempo a esta parte, es la de El amo del mar, novela del vizconde de Vogüe, que ha puesto en castellano D. José Plá y editado la casa Maucci de Barcelona”. Con todo, la traducción de José Pla no se salva de sus críticas.
} 
asimismo en tomos en octavo. Así lo subrayaba la noticia bibliográfica aparecida, entre otros, en La Rioja: diario político (10-III-1898):

Parecía imposible que después de la «Pequeña Enciclopedia Electromecánica» de Graffigny se pudiesen escribir libros distintos á éstos sobre electricidad; no sólo se han escrito, sino que la librería editorial Bailly Bailliere é hijos no ha tenido reparo en publicarlos, pues en lugar de perjudicar la venta de los de Graffigny, son los nuevos libros un complemento de aquéllos.

De hecho, la Pequeña Enciclopedia Electromecánica se publicitó en las últimas páginas de los volúmenes de la Biblioteca Completa de Electricidad y, una vez finalizada la colección, ambas se presentaron conjuntamente en la publicidad incluida en otras publicaciones. Sus volúmenes se vendieron incluso al mismo precio: los encuadernados en rústica, a 1,5 pesetas, y los encuadernados en tela -en este caso de color granate, con caracteres dorados-, a 2 pesetas. Solo el último de ellos, como se verá después, tenía un precio más elevado.

Como había ocurrido con la obra de Graffigny, la aparición de los sucesivos volúmenes de la colección de O'Conor Sloane se acompañó de una importante campaña de difusión -en esta ocasión si cabe más intensa-, en forma de reseñas y notas bibliográficas que se reprodujeron sin apenas cambios en las páginas de diferentes revistas de la época, así como en los principales diarios nacionales y regionales. Gracias a ellas se puede conocer con exactitud el orden y la fecha de aparición de los distintos volúmenes de la colección, cuyos principales datos se recogen en la figura 3:

\begin{tabular}{|c|c|c|c|}
\hline Título & $1 .^{\text {a }}$ ed. & $\begin{array}{l}\text { Tiradas y } \\
\text { ediciones }\end{array}$ & Obs. \\
\hline $\begin{array}{l}\text { 1. La electricidad simplificada } \\
\text { Teoría y práctica de la electricidad. } \\
\text { Examen popular de la teoría de la } \\
\text { electricidad y de sus aplicaciones á } \\
\text { los usos de la vida }\end{array}$ & $\begin{array}{l}\text { Marzo } 1898 \\
\text { (trad. de } \\
\text { la 2. }{ }^{\mathrm{a}} \text { ed. } \\
\text { inglesa) }\end{array}$ & $\begin{array}{l}1899\left(3 .^{a} \text { ti- }\right. \\
\text { rada }), 1903, \\
1906\left(9 .^{\text {a }} \text { ti- }\right. \\
\text { rada })\end{array}$ & 142 págs. \\
\hline $\begin{array}{l}\text { 2. Aritmética de la electricidad } \\
\text { Manual de cálculo de la electricidad } \\
\text { por métodos aritméticos. }\end{array}$ & $\begin{array}{l}\text { Marzo } 1898 \\
\text { (trad. de } \\
\text { la } 4 .^{\mathrm{a}} \text { ed. } \\
\text { inglesa) }\end{array}$ & $\begin{array}{l}1899\left(4 .^{\mathrm{a}} \text { ti- }\right. \\
\text { rada), } 1903\end{array}$ & 144 págs. \\
\hline
\end{tabular}




\begin{tabular}{|c|c|c|c|}
\hline Título & $10^{a}$ ed. & $\begin{array}{l}\text { Tiradas y } \\
\text { ediciones }\end{array}$ & Obs. \\
\hline $\begin{array}{l}\text { 3. Cómo se forma un buen electricista } \\
\text { Estudios, métodos de trabajar, campo } \\
\text { de operaciones, moral de la profesión }\end{array}$ & $\begin{array}{l}\text { Abril } 1898 \\
\text { (trad. del } \\
\text { inglés) }\end{array}$ & 1899 & 160 págs. \\
\hline $\begin{array}{l}\text { 4. La electricidad para todos } \\
\text { Construcción de pilas, imanes, elec- } \\
\text { tromotores, varios juguetes y dinamos }\end{array}$ & $\begin{array}{l}\text { Abril } 1898 \\
\text { (trad. de } \\
\text { la } 3 .^{a} \text { ed. } \\
\text { inglesa) }\end{array}$ & 1899,1906 & 142 págs. \\
\hline $\begin{array}{l}\text { 5. Diccionario práctico de electric- } \\
\text { idad } \\
\text { Colección de términos y expresiones } \\
\text { que se emplean en electricidad teórica } \\
\text { y aplicada }\end{array}$ & $\begin{array}{l}\text { Agosto } 1898 \\
\text { (trad. de } \\
\text { la 2. }{ }^{\text {a ed. }} \\
\text { inglesa) }\end{array}$ & $\begin{array}{l}1903,1911\left(6 .^{\mathrm{a}}\right. \\
\text { tirada) } \\
1918\left(2 .^{\mathrm{a}} \mathrm{ed} .\right)\end{array}$ & $\begin{array}{l}\text { 1.: }: 560 \\
\text { pp. } \\
\text { 2.: }: 633 \\
\text { pp. }\end{array}$ \\
\hline
\end{tabular}

Tabla 3. Volúmenes de la edición española de la Biblioteca Completa de Electricidad

De nuevo, las referencias a la presencia de grabados en sus páginas, a la claridad de la exposición y a su módico precio se convirtieron en un lugar común en las reseñas que daban noticia de la aparición de los sucesivos volúmenes ${ }^{25}$. Todo ello no hacía sino incidir en su carácter práctico y popular, que se adelantaba ya en el subtítulo de La electricidad simplificada.

Creemos inútil elogiar estas obritas, que por su módico precio [...] están al alcance de todas las fortunas; sólo nos concretaremos á decir que las juzgamos utilísimas, no sólo para el que se ocupa de electricidad, sino para todo el mundo (Heraldo de Madrid, 2-III-1898: 3) ${ }^{26}$.

${ }^{25}$ Solo en las páginas de Madrid Científico (n. ${ }^{\circ}$ 183, 1898: 1429-1430), en un artículo firmado por Chung, se referían ciertos problemas de orden terminológico: "La exposición es clara, pero á veces no muy precisa; así parece que se confunde la diferencia de carga con la diferencia de tensión y con la fuerza electro-motriz, que son cosas muy distintas; se dice también que el trabajo eléctrico se mide en watts y algunas otras cosillas por el estilo [...]. De la traducción nada tenemos que decir sino es que sería conveniente que en libros españoles no se usaran palabras que como selfinducción, voltage, conductancia, etcétera, son tan dudosamente castellanas".

${ }^{26}$ Esta breve reseña con motivo de la aparición de los dos primeros tomos, con algunas modificaciones, apareció en La Correspondencia de España (n. ${ }^{\circ}$ 14641, 5-III-1898), La Época (n. ${ }^{\circ}$ 17150, 6-III-1898: 4), El Globo (n. ${ }^{\circ} 8137,6$ 6-III-1898: 3), El Imparcial (8-III-1898: 3), El Cantábrico (n. ${ }^{\circ}$ 1048, 9-III-1898), El Diario de Murcia (n. ${ }^{\circ} 7599$, 
Estas últimas palabras recuerdan a las que se habían dedicado a la Pequeña Enciclopedia Electromecánica, "cuya lectura no requiere estudios especiales [...] y ayudará poderosamente en sus trabajos á cuantos estudien alguna aplicación eléctrica o mecánica", según se podía leer en el diario liberal El Pallaresa (12-V-1897: 2). No en vano, al igual que aquella, se presentaba como una obra destinada a la instrucción de las clases populares, que habían asistido a la irrupción de la electricidad en los establecimientos públicos y privados, en los hogares y en las calles de las ciudades españolas, y que veían en la joven industria eléctrica un vasto campo de oportunidades laborales:

Nuestros lectores conocen ya la colección, titulada: Pequeña Enciclopedia electromecánica, de utilidad inapreciable para cuantos deseen conocer la industria eléctrica [...], y como si eso no bastase para los propósitos de los editores, han emprendido la publicación de las obras de T. O'Conor Sloane, en las que se hermana la teoría con la práctica [...] (Industria e Invenciones, 26-III-1898: 11).

Es evidente que la formación técnica necesaria para desempeñarse profesionalmente en ese ámbito no estaba al alcance de todas las economías, de modo que la editorial Bailly-Baillière presentó la Biblioteca Completa de Electricidad -también la colección de Graffigny- como una alternativa sencilla y asequible para cuantos no podían seguir los estudios reglados. En este sentido, resulta revelador el siguiente fragmento, extraído de la noticia bibliográfica aparecida en las páginas de distintos periódicos y revistas coincidiendo con la publicación del tercer y cuarto volumen de la colección:

Llegar á ser ingenieros electricistas es la ambicion de millares de jóvenes y hombres de edad, pero no todos pueden hacer los cuantiosos gastos ni disponer de los tres ó cuatro años necesarios para esta carrera; sin embargo, puede llegarse á ser ingeniero electricista sin hacer tales gastos, y esta obra está destinada á dicho objeto, sin los sacrificios que

9-III-1898), La Rioja (10-III-1898), Diario de Córdoba (n. $\left.{ }^{\circ} 14032,12-I I I-1898\right), ~ R e-$ vista Minera, Metalúrgica y de Ingeniería (n. ${ }^{\circ}$ 1673, 16-III-1898: 107), La Ilustración Nacional (t. XVI, n. ${ }^{o}$ 8, 18-III-1898) y Blanco y Negro (26-III-1898: 20), entre otros. 
ordinariamente hay que hacer para conseguirlo (Diario de Murcia, $\mathrm{n}^{\circ}$ 7643, 23-IV-1898) ${ }^{27}$.

Las reiteradas alusiones a lo accesible de la obra, "la más sencilla que hasta ahora se ha publicado sobre electricidad", cuyos cálculos se reducían a "reglas sencillísimas", hacían el resto. También había una alusión explícita a los aficionados, a quienes se destinaba el volumen $4, L a$ electricidad para todos -la Pequeña Enciclopedia Electromecánica se cerraba con un tomo con este mismo título-, con cuya ayuda, señalaba la citada reseña, "podrán construir con sus propias manos gran número de piezas de aparatos eléctricos, que á la vez que les sirven de instrucción puede tambien servirles de recreo".

Una mención aparte merece el último volumen de la colección, correspondiente al Diccionario práctico de electricidad, subtitulado Colección de términos y expresiones que se emplean en electricidad teórica y aplicada ${ }^{28}$, que adquirió entidad propia desde su misma publicación, tanto por su contenido como por su mayor extensión, pues constaba de 560 páginas $^{29}$; de hecho, a diferencia de los anteriores títulos, se vendió a 13,50 pesetas en rústica y a 15 pesetas en tela. No en vano, el repertorio de O'Conor Sloane se convirtió en el segundo diccionario de electricidad en español, después del Diccionario de electricidad y magnetismo (1893) de Julien Lefèvre, publicado también por BaillyBaillière, como recordaba la prensa, que aprovechó la ocasión para des-

\footnotetext{
${ }^{27}$ De nuevo, con algunas alteraciones y recortes, esta extensa reseña de la obra se reprodujo en otros periódicos y revista con escaso margen de tiempo: El Cantábrico (n. ${ }^{\circ}$ 1103, 4-V-1898), La Correspondencia de España (n. ${ }^{\circ}$ 14702, 5-V-1898), La Región Extremeña (n. $\left.{ }^{\circ} 3769,6-\mathrm{V}-1898\right)$, Revista Minera, Metalúrgica y de Ingeniería (n. ${ }^{\circ} 1680$, 8-V-1898: 167), Industria e Invenciones (14-V-1898: 185), La Ilustración Nacional (t. XVI, n. $\left.{ }^{\circ} 14,20-\mathrm{V}-1898\right)$, etc.

${ }^{28}$ El subtítulo de la edición americana, ligeramente distinto del de la traducción española, era más explícito en lo que respecta a su carácter popular: A popular dictionary of words and terms used in the practice of electrical engineering. También lo era la edición londinense de 1898: A popular encyclopaedia of words and terms used in the practice of electrical engineering. La traducción española se realizó a partir de la segunda edición americana, publicada en 1897.

${ }^{29}$ A propósito de la edición inglesa del diccionario de O’Conor Sloane, en la Revista Contemporánea (t. CXI, vol. V, 15-IX-1898) se apuntaba lo siguiente: "La primera copiosa edición de este Diccionario se agotó en pocos años, y seguramente ocurrirá lo mismo con la segunda, porque hoy se ha generalizado tanto la electricidad que se hace indispensable el constante manejo del libro compuesto por el señor Sloane”.
} 
tacar de nuevo la importante "obra de vulgarización técnica y científica que ha emprendido [la editorial madrileña] y que en nuestro país es más necesaria y útil que en parte alguna" (Revista Minera, Metalúrgica y de Ingeniería, . $^{\circ}$ 1696, 8-IX-1898: 295):

Esta casa, en su idea constante de hacer grandes electricistas á nuestros jóvenes y de ayudar poderosamente en sus conocimientos á los ya sabios, no repara en circunstancias ni gastos. Primero publicó el Diccionario de Electricidad, de Lefèvre; luego la importante Enciclopedia Electromecánica, de Graffigny, y, en fin, la Biblioteca de Electricidad, de Sloane [...]. El tomo V es un diccionario de electricidad, y la casa editorial, á pesar de tener ya el de Lefèvre, que alcanzó un éxito merecido, no ha vacilado en imprimir este nuevo diccionario, que por su índole lo hace diferente del otro (El Globo, n. $\left.{ }^{\circ} 8306,25-V I I I-1898: 3\right)^{30}$.

En efecto, las diferencias entre el diccionario de Lefèvre y el de O’Conor Sloane eran notables. Así, mientras que el repertorio francés se presentaba como un volumen de considerables dimensiones $(28 \times 20$ cm y 1.068 páginas), el repertorio inglés, a pesar de sus más de quinientas páginas, seguía siendo -como los restantes tomos de la Biblioteca Completa de Electricidad - un manual ligero y de fácil consulta. Por esa misma razón, así como las ilustraciones de Lefèvre (1.125 en todo el volumen) tenían un marcado carácter ornamental, las que acompañan a los artículos del diccionario de O'Conor Sloane (381 en total) se distinguían por su sencillez y esquematismo, y por ello "ayudan poderosamente á su mejor comprensión" (ibid.). Por otra parte, mientras que el diccionario francés ofrecía 1.315 artículos, el repertorio inglés ofrecía 2.832 -incluidas en ambos casos las remisiones-, fruto de la mayor extensión de los artículos de Lefèvre ${ }^{31}$.

\footnotetext{
${ }^{30}$ Estas mismas palabras se reprodujeron, entre otros, en El Diario de Murcia (n. ${ }^{\circ} 7767$, 25-VIII-1898), La Época (n. ${ }^{\circ}$ 17325, 30-VIII-1898: 3), El Heraldo de Madrid (31VIII-1898: 3) y El Liberal (8-IX-1898: 4). También se dio noticia de su publicación en La Rioja, diario político (n. $\left.{ }^{\circ} 2941,25-V I I I-1898\right)$, La Correspondencia Alicantina (n. ${ }^{\circ}$ 2101, 26-VIII-1898), Diario de Córdoba (n. $\left.{ }^{\circ} 14189,26-V I I I-1898\right)$, La Correspondencia de España (n. $\left.{ }^{\circ} 14817,28-V I I I-1898 ; ~ n .^{\circ} 14818,29-V I I I-1898\right)$, Blanco y Negro (3IX-1898: 18), Revista Minera, Metalúrgica y de Ingeniería (n. ${ }^{\circ}$ 1696, 8-IX-1898: 295) y Revista Contemporánea (15-IX-1898), que ofrecieron sendas reseñas del diccionario. ${ }^{31}$ Para más detalles sobre las características de ambos diccionarios, así como sobre la nomenclatura incluida en ellos y su tratamiento lexicográfico, véase Madrona \& Moreno (2004) y Moreno Villanueva (2012: 144-174).
} 
Esas distintas características externas e internas acercaban el diccionario francés al género de las enciclopedias o diccionarios enciclopédicos, y el inglés, al de los diccionarios terminológicos; una distinción en la que O'Conor Sloane incidía en el prólogo a la primera edición americana $^{32}$ :

La ciencia se ha desarrollado de tal modo, que lo que hoy puede llamarse estrictamente diccionario hubiera bastado hace pocos años para una enciclopedia. Una enciclopedia de electricidad sería por consiguiente una obra muy voluminosa. [...] un diccionario con buenas definiciones, y reducido á los límites más estrechos por medio de la enunciación de los sinónimos, y en el que las innumerables referencias mutuas sean trasladadas á un índice conciso, será bastante más que lo que ordinariamente se entiende por un diccionario.

En efecto, tras el cuerpo central del repertorio (pp. 1-535), O'Conor Sloane ofrece un índice final (pp. 537-560) en el que se incluyen tanto sus lemas como los sinónimos que aparecen al pie de los respectivos artículos, pues los conceptos se definen una sola vez en el diccionario: "Si el lector busca la definición de uno de estos sinónimos, consulte el índice y verá la página en que está la información que se desea"33.

Todo ello, en fin, hacía del diccionario "un libro conciso y práctico de referencias", "un libro dedicado á la práctica y al manejo diarios" (Revista Minera, Metalúrgica y de Ingeniería, n. ' 1696, 8-IX-1898: 295). Fruto de la buena acogida que tuvo, en enero de 1918, BaillyBaillière publicó una segunda edición, ampliada y revisada por el ingeniero José María Jiménez Quintana, de 633 páginas $^{34}$. El volumen, que se vendió a 8,50 pesetas en rústica y a 10 pesetas encuadernado en tela, se presentaba en La Energía Eléctrica (10-II-1918: 11) como "no sólo necesario al modesto instalador electricista, sino imprescindible al más eminente ingeniero", algo en lo que ya se había insistido con motivo de la publicación de su primera edición en español:

\footnotetext{
${ }^{32}$ Cito por la edición española del diccionario.

${ }^{33}$ Pese a estas indicaciones, a lo largo del diccionario se pueden localizar diversos errores, que dan lugar, por una parte, a pistas perdidas y, por otra, a la definición de dos palabras sinónimas bajo sus respectivos artículos.

${ }^{34}$ Como los volúmenes anteriores, la publicación de esta segunda edición se publicitó, entre otros, en El Heraldo de Madrid (16-I-1918: 6, 23-I-1918: 4, 27-I-1918: 6, 4-II1918: 6, 6-II-1918: 6, 7-IV-1918: 6) y El Día (23-I-1918: 4).
} 
El objeto del autor al publicar este libro [el Diccionario Práctico de Electricidad] es el que hace mucho tiempo se perseguía y con insistencia pedían todas aquellas personas que se dedican al estudio y práctica de la electricidad, así como también las profanas que tienen relación con esta importante ciencia. (La Rioja, diario político, n. ${ }^{\circ} 2941$, 25-VIII-1898) $)^{35}$

En última instancia, el diccionario de O'Conor Sloane, con el que se completaba la Biblioteca Completa de Electricidad, además de facilitar el acceso a los conocimientos teóricos y prácticos necesarios para desempeñarse en el ámbito de la electricidad práctica, contribuía a difundir y, en cierto modo, también a fijar la terminología eléctrica tanto entre los ya iniciados en la materia como entre el público profano.

\section{Conclusión}

La Pequeña Enciclopedia Electromecánica y la Biblioteca Completa de Electricidad constituyen un buen ejemplo del papel que desempeñaron las colecciones de divulgación científica y técnica entre finales del siglo XIX y principios del siglo XX. En un contexto favorable a la instrucción popular y ayudadas por los avances técnicos que habían introducido profundas transformaciones en el sector, las empresas editoriales apostaron por la publicación de este género de bibliotecas, conscientes de la existencia de un amplio público interesado por los avances de la técnica y sus aplicaciones.

Por lo que respecta a la electricidad, ese público incluía ingenieros técnicos especializados, constructores, electricistas prácticos, obreros empleados en la naciente industria eléctrica, jóvenes en formación e, incluso, usuarios finales; de ahí que el texto de los volúmenes que conforman las colecciones estudiadas se mueva entre la instrucción y el entretenimiento. Por esa misma razón, aunque en las portadas y prólogos de esas obras se alude de manera habitual a su utilidad, su carácter práctico y su lenguaje claro, sencillo y conciso con el propósito de ganar

\footnotetext{
${ }^{35}$ Estas mismas palabras, con ligeras modificaciones, se pudieron leer, entre otros, en La Correspondencia Alicantina (n. ${ }^{\circ}$ 2101, 26-VIII-1898), Diario de Córdoba (n. ${ }^{\circ}$ 14189, 26-VIII-1898), El Cantábrico (n. ${ }^{\circ}$ 1218, 27-VIII-1898), La Región Extremeña

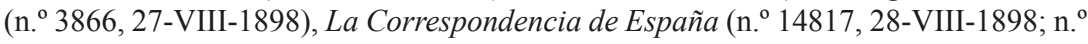
14818, 29-VIII-1898) e Industria e Invenciones (3-IX-1898: 6).
} 
lectores, no es menos cierto que, al mismo tiempo, esas colecciones se perfilaban "como un contrapoder a la ciencia académica de los profesionales" (Nieto-Galan 2011: 18).

La buena acogida de que disfrutaron estos manuales evidencia el acierto de la estrategia comercial seguida por las casas editoriales. En este sentido, se constata un paralelismo evidente entre la forma de proceder de las editoriales francesa y americana y la editorial Bailly-Baillière e Hijos, que hizo valer su posición en el mercado del libro español para introducir en él un género de colecciones que contaba ya con un notable recorrido en otros países, sobre todo en Francia.

Esta última circunstancia, unida a la escasez de obras de autor español dedicadas al estudio de la electricidad y sus aplicaciones prácticas, determinó que Bailly-Baillière optara por traducir las dos colecciones estudiadas. De hecho, la gran aceptación que tuvieron llevó a la editorial madrileña a publicar, entre otras, la Pequeña Enciclopedia de Agricultura, de A. Larbatrier; la Pequeña Enciclopedia Práctica de Construcción, de L. A. Barré; la Pequeña Enciclopedia de Química Industrial, de F. Billon; y la Enciclopedia del Fotógrafo Aficionado, de G. Brunel. Otras editoriales intentaron seguir sus pasos; fue el caso de Montaner y Simón, Manuel Soler o José Gallach, estas últimas impulsoras de los conocidos Manuales Soler-Gallach (Nieto-Galan, 2008; Moreno Villanueva, 2017b).

Naturalmente, la difusión de la electricidad práctica entre un amplio público supuso también la divulgación de la terminología asociada; por ello, se constata un notable esfuerzo por facilitar al acceso a ella. En el caso de las obras de Graffigny y O'Conor Sloane, ese esfuerzo se manifestó en el recurso a la cursiva para destacar los numerosos tecnicismos incluidos en el texto y, sobre todo, en la inclusión de sendos vocabularios de voces técnicas: en el primer caso, en forma de pequeño apéndice al último de sus tomos; en el segundo, en forma de un extenso diccionario que constituyó uno de sus volúmenes y que, por esa misma razón, adquirió entidad propia.

Por todo ello, más allá de su valor estrictamente científico y de su utilidad social, es preciso subrayar el papel que este género de obras desempeñó en la articulación de un nuevo discurso divulgativo, o vulgarizador, así como en relación con la difusión de nuevos términos, y reivindicar el justo lugar que le corresponde en la historia de la traducción científica y técnica. 


\section{Bibliografía}

Alayo, Joan Carles. 2003. L'electricitat a Espanya en els segles XVIII i XIX. Una anàlisi a partir de la bibliografia histórica. En Batlló, Josep; Bernat, Pasqual \& Puig, Roser (coords.) Actes de la VII Trobada d'Història de la Ciència i de la Tècnica. Barcelona: Societat Catalana d'Història de la Ciencia i de la Tècnica, 433-438.

Alayo, Joan Carles. 2007. L'electricitat a Catalunya. De 1875 a 1935. Lleida: Pagès Editors.

Alayo, Joan Carles \& Sánchez Miñana, Jesús. 2011. La introducción de la técnica eléctrica. En: Silva Suárez, Manuel (ed.) Técnica e ingeniería en España. VI. El Ochocientos. De los lenguajes al patrimonio. Zaragoza: Real Academia de Ingeniería / Institución Fernando el Católico / Prensas Universitarias de Zaragoza, 650-699.

Béguet, Bruno. 1990. La science pour tous. Sur la vulgarisation scientifique en France de 1850 à 1914. París: Bibliothèque du Conservatoire National des Arts et Métiers.

Botrel, Jean-François. 1988. La diffusion du livre en Espagne (1868-1914). Madrid: Casa de Velázquez.

Botrel, Jean-François. 1993. Libros, prensa y lectura en la España del siglo XIX. Madrid: Fundación Germán Sánchez Ruipérez.

Cano Pavón, José Manuel \& López-Cepero, José M. 2002. La física en las escuelas industriales españolas en la época isabelina (1850-1868). Llull 25: 595-620.

Cano Pavón, José Manuel. 2007. El Real Instituto Industrial de Madrid y las escuelas periféricas. En Silva, Manuel (ed.) Técnica e ingeniería en España. V. El Ochocientos. Profesiones e instituciones civiles. Zaragoza: Real Academia de Ingeniería / Institución Fernando el Católico / Prensas Universitarias de Zaragoza, 295-350.

Ferran Boleda, Jordi. 2013. Els públics de l'electricitat a Catalunya (19291936): de la Font Màgica de Montjuïc a la difusió dels electrodomèstics (Tesis Doctoral). Universitat Autònoma de Barcelona.

Gourevitch, Danielle \& Vincent, Jean-François (eds.). 2006. J.-B. Baillière et fils, éditeurs de medicine. París: De Boccard Édition-Diffussion.

Lusa Monforte, Guillermo. 2003. La Escuela de Ingenieros Industriales de Barcelona y la introducción de la electricidad industrial en España (18721899). En Batlló, Josep; Bernat, Pasqual \& Puig, Roser (coords.) Actes de la VII Trobada d'Història de la Ciència i de la Tècnica. Barcelona: Societat Catalana d'Història de la Ciencia i de la Tècnica, 373-384.

Lusa Monforte, Guillermo. 2007. La Escuela de Ingenieros Industriales de Barcelona. En Silva, Manuel (ed.) Técnica e ingeniería en España. 
V. El Ochocientos. Profesiones e instituciones civiles. Zaragoza: Real Academia de Ingeniería / Institución Fernando el Católico / Prensas Universitarias de Zaragoza, 351-394.

Madrona, Alicia \& Moreno Villanueva, José Antonio. 2004. Los primeros diccionarios de electricidad en español: el Diccionario de electricidad y magnetismo (1893) de Lefèvre y el Diccionario práctico de electricidad (1898) de O'Conor Sloane. En Battaner, Paz \& DeCesaris, Janet (eds.) De Lexicografia. Barcelona: IULA-UPF, 605-617.

Martínez Martín, Jesús A. 2001. Historia de la edición en España. 1836-1936. Madrid: Marcial Pons.

Moreno González, Antonio. 1988. Una ciencia en cuarentena: sobre la física en la universidad y otras instituciones académicas desde la Ilustración hasta la crisis finisecular del XIX. Madrid: CSIC.

Moreno Villanueva, José Antonio. 2012. Formación y desarrollo del léxico de la electricidad en español (siglos XVIII-XIX). (Tesis Doctoral). Tarragona: Universitat Rovira i Virgili.

Moreno Villanueva, José Antonio. 2015. Los manuales de procedencia francesa en la enseñanza y difusión de la física eléctrica en España a lo largo del siglo XIX. En Pinilla, Julia \& Lépinette, Brigitte (eds.) Traducción y difusión de la ciencia y la técnica en España (s. XVI-XIX). Valencia: Universitat de València, 277-294.

Moreno Villanueva, José Antonio. 2017a. Los Manuales Gallach: materiales para la historia de la lexicografía especializada. En Garriga, Cecilio; Sariego, Ignacio \& Gutiérrez Cuadrado, Juan. El diccionario en la encrucijada: de la sintaxis y la cultura al desafio digital. Santander: Escuela Universitaria de Turismo Altamira, 647-664.

Moreno Villanueva, José Antonio. 2017b. Bailly-Baillière y la divulgación de la técnica: la Pequeña Enciclopedia Electromecánica. En Pinilla, Julia \& Lépinette, Brigitte (eds.) Reconstruyendo el pasado de la traducción II. Granada: Comares, 119-131.

Nieto-Galan, Agustí. 2008. El 1libre de divulgació científica a la Barcelona de finals de segle XIX: autors, editorials, públics. En Vélez, Pilar (ed.) L'exaltació del llibre al Vuitcents. Art, indústria i consum a Barcelona. Barcelona: Biblioteca de Catalunya, 201-220.

Nieto-Galan, Agustí. 2011. Los públicos de la ciencia. Expertos y profanos a través de la historia. Madrid: Fundación Jorge Juan / Marcial Pons.

Ramón, Javier \& Silva, Manuel. 2007. El Real Conservatorio de Artes (18241887), cuerpo facultativo y consultivo auxiliar en el ramo de industria. En Silva, Manuel (ed.) Técnica e ingeniería en España. V. El Ochocientos. Profesiones e instituciones civiles. Zaragoza: Real Academia 
de Ingeniería / Institución Fernando el Católico / Prensas Universitarias de Zaragoza, 235-294.

Riera Clement, Cristina \& Riera Palmero, Juan. 2005. El influjo médico extranjero en España (1850-1900). Llull 26: 503-524.

Sánchez Miñana, Jesús. 2006. Las primeras aplicaciones de la electricidad en Barcelona en torno a 1850. Quaderns d'Història de l'Enginyeria 7: 115-195.

Sánchez Sánchez, Isidro. 1999. Las luces del 98. Sociedades eléctricas en la España finisecular. En Sánchez Sánchez Sánchez, Isidro \& Villena Espinosa, Rafael (coords.) Sociabilidad fin de siglo. Espacios asociativos en torno a 1898. Cuenca: Ediciones de la Universidad de Castilla-La Mancha, 151-224.

Sánchez Vigil, Juan Miguel \& Olivera Zaldua, María. 2014. La editorial Gallach y su contribución a la industria cultural española. Recuperación y análisis de su catálogo. Información Bibliotecológica 28: 51-83.

Silva, Manuel (ed.) 2007. Técnica e ingeniería en España V. El Ochocientos. Profesiones e instituciones civiles. Zaragoza: Real Academia de Ingeniería / Institución Fernando el Católico / Prensas Universitarias de Zaragoza.

Simon Castel, Josep. 2006. La famille Baillière et l'introduction du Traité de Physique de Ganot en Angleterre. En Gourevitch, Danielle \& Vincent, Jean-François (eds.) J.-B. Baillière et fils, éditeurs de medicine. París: De Boccard Édition-Diffussion, 193-204.

Simon Castel, Josep. 2007. Comunicando la física en la Europa del siglo XIX: el manual de Ganot y los oficios del libro. En Herran Corbacho, Néstor; Simon Castel, Josep; Guillem-Llobat, Ximo; Lanuza, Tayra M. C.; Ruiz Castell, Pedro \& Navarro, Jaume (coords.) Synergia. Primer Encuentro de Jóvenes Investigadores en Historia de la Ciencia. Madrid: Consejo Superior de Investigaciones Científicas, 29-48.

Simon Castel, Josep. 2010. The Baillières. Franco-British book trade and the transit of knowledge. In Fox, Robert \& Joly, Bernard (eds.) Franco-British interactions in science since the seventeenth century. Londres: College Publications, 243-262. 